\title{
The mammary stem cell conundrum: is it unipotent or multipotent?
}

\author{
Purna A Joshi, ${ }^{1,2}$ and Rama Khokha ${ }^{* 1-3}$
}

\begin{abstract}
Exploring the normal biology and regulation of stem cells has the promise to yield insights into the etiological roots and survival of breast cancer cells. Many studies have supported the existence of a multipotent mammary stem cell that regenerates all aspects of glandular development. However, Van Keymeulen and colleagues (2011) illustrated the presence of lineage-restricted unipotent stem cells that self-renew and collaborate in postnatal mammary development, whereas multipotent stem cells were found only during embryonic mammogenesis. This prompts a re-evaluation of currently accepted mammary stem cell dynamics and conceivably its impact on the evolution of different breast cancer subtypes.
\end{abstract}

The capacity of the mammary gland for rapid growth and regeneration is attributed to mammary stem cells (MaSCs). Mammopoiesis initiates in the embryo but the majority of glandular development occurs during puberty. Recurrent reproductive cycles elicit transient but significant alveolar epithelial expansion, whereas pregnancy involves heightened lobuloalveologenesis and lactational differentiation. Deome and colleagues [1] were the first to show that tissue transplants from several portions of the gland form an entire gland in vivo in epithelium-divested fat pads, suggesting the existence of MaSCs. Transplantation of tissue fragments or dispersed cells has since become a routine functional assay in the mammary gland field. Limiting-dilution transplants of cells have demonstrated the capacity of single cells to clonally expand to form a functional mammary gland and self-renew in serial transplants, supporting the existence of a multipotent MaSC [2].

*Correspondence: rkhokha@uhnresearch.ca

Ontario Cancer Institute, 610 University Avenue, Toronto, ON, M5G 2M9, Canada Full list of author information is available at the end of the article
Bilayered mammary ducts are composed of inner luminal epithelial and outer basal/myoepithelial cells. When mammary cells are purified by fluorescenceactivated cell sorting (FACS) using surface markers that segregate luminal $\left(\mathrm{CD} 24^{\mathrm{med} /}+\mathrm{CD} 49 \mathrm{f}^{1 \mathrm{lo}} / \mathrm{CD} 29^{\mathrm{lo}}\right)$ and basal $\left(\mathrm{CD} 24^{\mathrm{med} /} \mathrm{CD} 49 \mathrm{f}^{\mathrm{hi}} / \mathrm{CD} 29^{\mathrm{hi}}\right)$ cells and are transplanted, basal cells generate robust functional mammary outgrowths whereas luminal cells lack this capacity $[3,4]$. Indeed, a single sorted basal cell has been shown to generate an entire mammary gland constituting ducts and alveoli and serially transplant in vivo. This illustrates the multipotent and self-renewal capacity of the basal MaSC. Injections of sorted/unsorted cells in limiting dilutions allow deductions of MaSC numbers in varying experimental conditions [5,6]. Interestingly, human breast stem cells capable of in vivo reconstitution were also found in basal cells (CD49f $\left.{ }^{+} \mathrm{EPCAM}^{\text {neg-low }}\right)$ but not in luminal cells [7].

Van Keymeulen and colleagues [8] reported the existence of lineage-restricted unipotent stem cells in both luminal and basal epithelial populations and challenged prevailing work on the contribution of multipotent MaSCs to postnatal gland development. The authors performed lineage-tracing experiments of embryonic, pubertal, adult, pregnant, and involuting mammary glands, employing K14-rtTA/TetO-Cre/Rosa-YFP and K5-CreER/Rosa-YFP mice for tracking basal cells and K8-CreER/Rosa-YFP and K18-CreER/Rosa-YFP mice for tracking luminal cells. Induction of K14-driven YFP in embryos led to labeling of both luminal and basal cells at puberty, implying that embryonic $\mathrm{K}^{+} 4^{+}$cells are multipotent. Postnatal YFP induction showed exclusive labeling of basal cells that clonally expanded during puberty and pregnancy. Similar results were obtained with $\mathrm{K} 5$-CreER, indicating that $\mathrm{K} 14^{+} / \mathrm{K}^{+}$cells are unipotent and do not contribute to luminal cell progeny. In contrast, YFP driven by K8/K18 luminal promoters labeled only luminal cells that clonally expanded in K8CreER mice but not in K18-CreER mice.

The differentiation potential of both epithelial lineages was determined by using transplantation assays. Unsorted cells from basal-specific Cre lines generated outgrowths in which $\mathrm{YFP}^{+}$cells were predominantly 
basal, although $\mathrm{YFP}^{+}$clones were also rarely seen in luminal cells. $\mathrm{YFP}^{+}$cells from the luminal-specific Cre line remained restricted to the luminal layer similar to that observed in the intact gland. This YFP chimerism was preserved in secondary grafts that the authors cite as evidence of self-renewal in these unipotent cells. When FACS-purified $\mathrm{YFP}^{+}$basal or luminal cells were transplanted, basal, but not luminal, cells were capable of reconstituting a mammary gland, recapitulating previous findings that originally defined multipotent basal MaSCs. In mixing experiments, reducing the luminal/basal cell ratio increased the potential of basal cells to generate luminal cells. The study argues that the experimental setting of the transplantation assay forces differentiation of basal MaSCs into both epithelial lineages while the intact gland relies on lineage-restricted unipotent stem cells.

Lineage-tracing experiments have proven valuable in uncovering new insights into stem cells in other tissue contexts such as the skin and intestine, and Van Keymeulen and colleagues [8] have harnessed this technique to address a fundamental question about stem cell differentiation potential. Their findings bring new perspective to the MaSC field. Previous work has adhered to transplantation as a reliable gold-standard stem cell assay. The lack of detection of a multipotent basal MaSC in lineagetracing experiments of the intact postnatal gland now thrusts scepticism on the contribution of basal multipotent stem cells to postnatal gland regeneration. Although the study broadens our conceptualization of the MaSC and raises questions about the relevance of transplantation as a functional stem cell readout, facets of this study warrant further discussion. Stem cells in other tissues such as the hematopoietic system have been shown to possess varying degrees of self-renewal, including short-term, long-term, and even intermediate repopulating potentials [9]. The basal and luminal unipotent cells were shown to maintain their chimerism in secondary grafts that the authors demonstrate to be selfrenewal but whether these cells have short- or long-term potential is unclear. Second, luminal cells, though unipotent, were unable to regenerate a gland independent of basal cells in transplantations, whereas basal cells possess this intrinsic property, implying a hierarchy of stem cell capacity. Luminal cells clearly rely on basal cells for cues that are likely paracrine to facilitate their unipotency in the intact gland. Furthermore, several groups have identified dual-positive cells $\left(\mathrm{K}_{1} 4^{+}\right.$with $\mathrm{K}^{+} /$ $\left.\mathrm{K} 18^{+} / \mathrm{K} 19^{+}\right)$in the human breast, suggesting the existence of bipotent progenitors and candidate stem cell zones [10-12]. Dual-positive cells, as well as markers that tag basal cells, have also been noted in situ in the murine gland within the luminal compartment of terminal end buds and alveoli $[13,14]$. Van Keymeulen and colleagues
[8] found $\mathrm{Lgr}^{+}$cells, an intestinal stem cell marker, predominantly in basal mammary cells but additionally in luminal cells. It is conceivable that these are indicative of a bipotent cell population but this possibility has been overlooked in their lineage-tracing experiments, perhaps because of low K14/K5 expression levels in dual-positive cells.

Identifying epithelial populations that have regenerative capacity and defining their extrinsic and intrinsic regulatory mechanisms are relevant to understanding not only the normal development but also the etiology of breast cancers in which transformed cells share properties akin to those of stem cells, notably self-renewal [15]. Given the heterogeneity of breast cancers, it is surmised that specific mammary cells are cells of origin for different cancer subtypes. It will be important to understand whether the likely targets of transformation are initially unipotent or multipotent and whether the mutation repertoire influences their differentiation potential and self-renewal capacity.

Abbreviations

FACS, fluorescence-activated cell sorting; MaSC, mammary stem cell.

Competing interests

The authors declare that they have no competing interests.

\section{Acknowledgments}

PAJ is supported by a fellowship from the Canadian Breast Cancer Foundation (CBCF), and RK is funded by grants from the CBCF and the Canadian Breast Cancer Research Alliance.

\section{Author details}

'Ontario Cancer Institute, 610 University Avenue, Toronto, ON, M5G 2M9, Canada. ${ }^{2}$ Department of Laboratory Medicine and Pathobiology, Faculty of Medicine, University of Toronto, Medical Sciences Building, 1 King's College Circle, 6th floor, Toronto, ON, M5S 1A8, Canada. ${ }^{3}$ Department of Medical Biophysics, University of Toronto, Ontario Cancer Institute, Princess Margaret Hospital, 610 University Avenue, Room 7-411, Toronto, ON, M5G 2M9, Canada.

Published: 16 March 2012

\section{References}

1. Deome KB, Faulkin LJ Jr., Bern HA, Blair PB: Development of mammary tumors from hyperplastic alveolar nodules transplanted into gland-free mammary fat pads of female C3H mice. Cancer Res 1959, 19:515-520.

2. Kordon EC, Smith $\mathrm{GH}$ : An entire functional mammary gland may comprise the progeny from a single cell. Development 1998, 125:1921-1930.

3. Shackleton M, Vaillant F, Simpson KJ, Stingl J, Smyth GK, Asselin-Labat ML, Wu L, Lindeman GJ, Visvader JE: Generation of a functional mammary gland from a single stem cell. Nature 2006, 439:84-88.

4. Stingl J, Eirew P, Ricketson I, Shackleton M, Vaillant F, Choi D, Li HI, Eaves CJ: Purification and unique properties of mammary epithelial stem cells. Nature 2006, 439:993-997.

5. Joshi PA, Jackson HW, Beristain AG, Di Grappa MA, Mote PA, Clarke CL, Stingl J, Waterhouse PD, Khokha R: Progesterone induces adult mammary stem cell expansion. Nature 2010, 465:803-807.

6. Badders NM, Goel S, Clark RJ, Klos KS, Kim S, Bafico A, Lindvall C, Williams BO, Alexander CM: The Wnt receptor, Lrp5, is expressed by mouse mammary stem cells and is required to maintain the basal lineage. PLoS One 2009, 4:e6594.

7. Eirew P, Stingl J, Raouf A, Turashvili G, Aparicio S, Emerman JT, Eaves CJ: A method for quantifying normal human mammary epithelial stem cells with in vivo regenerative ability. Nat Med 2008, 14:1384-1389.

8. Van Keymeulen A, Rocha AS, Ousset M, Beck B, Bouvencourt G, Rock J, 
Sharma N, Dekoninck S, Blanpain C: Distinct stem cells contribute to mammary gland development and maintenance. Nature 2011, 479:189-193.

9. Benveniste P, Frelin C, Janmohamed S, Barbara M, Herrington R, Hyam D, Iscove NN: Intermediate-term hematopoietic stem cells with extended but time-limited reconstitution potential. Cell Stem Cell 2010, 6:48-58.

10. Villadsen R, Fridriksdottir AJ, Ronnov-Jessen L, Gudjonsson T, Rank F, LaBarge MA, Bissell MJ, Petersen OW: Evidence for a stem cell hierarchy in the adult human breast. J Cell Biol 2007, 177:87-101.

11. Pasic L, Eisinger-Mathason TS, Velayudhan BT, Moskaluk CA, Brenin DR, Macara IG, Lannigan DA: Sustained activation of the HER1-ERK1/2-RSK signaling pathway controls myoepithelial cell fate in human mammary tissue. Genes Dev 2011, 25:1641-1653.

12. Clayton $\mathrm{H}$, Titley I, Vivanco M: Growth and differentiation of progenitor/ stem cells derived from the human mammary gland. Exp Cell Res 2004, 297:444-460.
13. Bai L, Rohrschneider LR: s-SHIP promoter expression marks activated stem cells in developing mouse mammary tissue. Genes Dev 2010, 24:1882-1892.

14. McCaffrey LM, Macara IG: The Par3/aPKC interaction is essential for end bud remodeling and progenitor differentiation during mammary gland morphogenesis. Genes Dev 2009, 23:1450-1460.

15. Al-Hajj M, Wicha MS, Benito-Hernandez A, Morrison SJ, Clarke MF: Prospective identification of tumorigenic breast cancer cells. Proc Natl Acad Sci U S A 2003, 100:3983-3988.

doi: $10.1186 /$ bcr3123

Cite this article as: Joshi PA, Khokha R: The mammary stem cell conundrum: is it unipotent or multipotent? Breast Cancer Research 2012, 14:305. 